\title{
Primary band-shaped spheroidal degeneration of the cornea: three cases from two consanguineous families
}

\author{
TETSUO HIDA,' KAZUTERU KIGASAWA,' ETSUKO TANAKA,' \\ SHINOBU AKIYA,' YUKIO TASHIRO, ${ }^{2}$ AND YASUHIRO HOSODA, ${ }^{2}$ \\ From the Departments of 'Ophthalmology and 'Pathology, Keio University, School of Medicine, Tokyo
}

SUMMARY Three cases of primary band-shaped spheroidal degeneration of the cornea from two consanguineous families are reported. In all cases vision deteriorated slowly, the deterioration beginning in childhood. Photophobia in childhood was also a common symptom, and two patients suffered from intermittent painful sensations. The occupational and environmental histories had no bearing on the cases. Histochemical study of the yellow, oily appearing deposits suggested that this material has a complex composition, with staining characteristics of both protein and acid-fast lipid.

Spheroidal degeneration of the cornea has been divided into two basic forms: primary and secondary.' The primary form is an aging process which affects only the peripheral cornea. The secondary form is constantly associated with other ocular pathology such as absolute glaucoma, phthisis bulbi, post-traumatic scars, and lattice corneal dystrophy. Climatic droplet keratopathy, ${ }^{2}$ which is found in certain geographic regions where the eyes are exposed to climatic extremes, is categorised in the latter form. In both of these types of spheroidal degeneration of the cornea familial occurrence is extremely rare. This report describes three cases of primary band-shaped spheroidal degeneration of the cornea from two consanguineous families.

\section{Case reports}

\section{CASE 1}

A 42-year-old female was referred to us for corneal transplant in January 1983 because of progressively decreasing vision and recurrent painful irritation in both eyes for the past three years. Her vision had been slowly deteriorating since childhood. She had also been very photophobic during her childhood. She had not been examined by an ophthalmologist

Correspondence to Tetsuo Hida, MD, Duke University Eye Center, Box 3082, Durham, North Carolina 27710, USA.

Reprint requests to Tetsuo Hida, MD, Department of Ophthalmology, Keio University School of Medicine, Shinanomachi 35, Shinjuku-ku, Tokyo 160, Japan. until 31 years of age, when she was diagnosed as having bilateral corneal degeneration.

Occupational and environmental histories contributed nothing to the case. Her parents were first cousins and one of her brothers had the same corneal
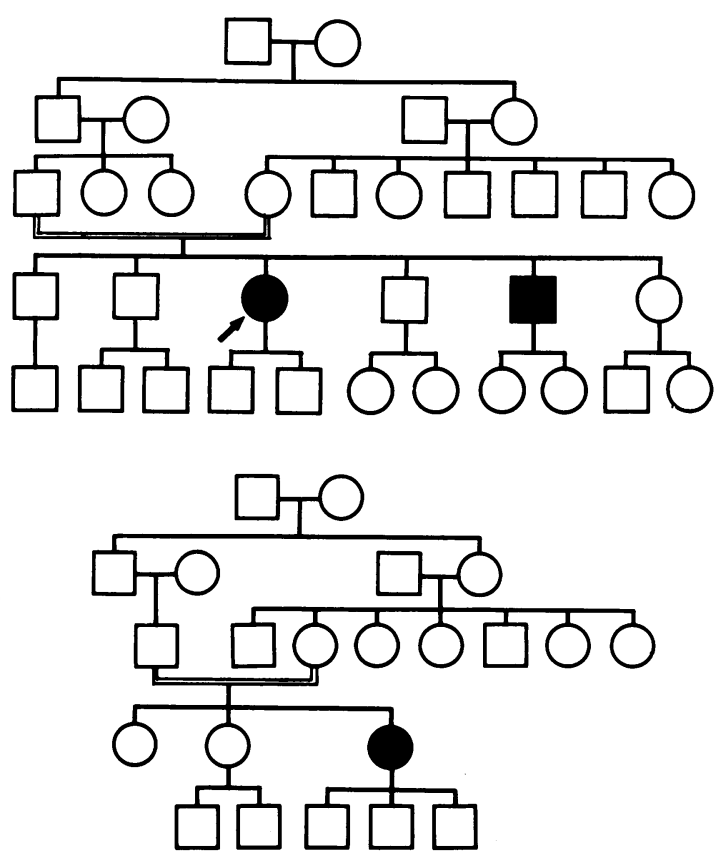

Fig. 1 Family trees showing consanguinity. Top, family of cases 1 and 2. Bottom, family of case 3. 


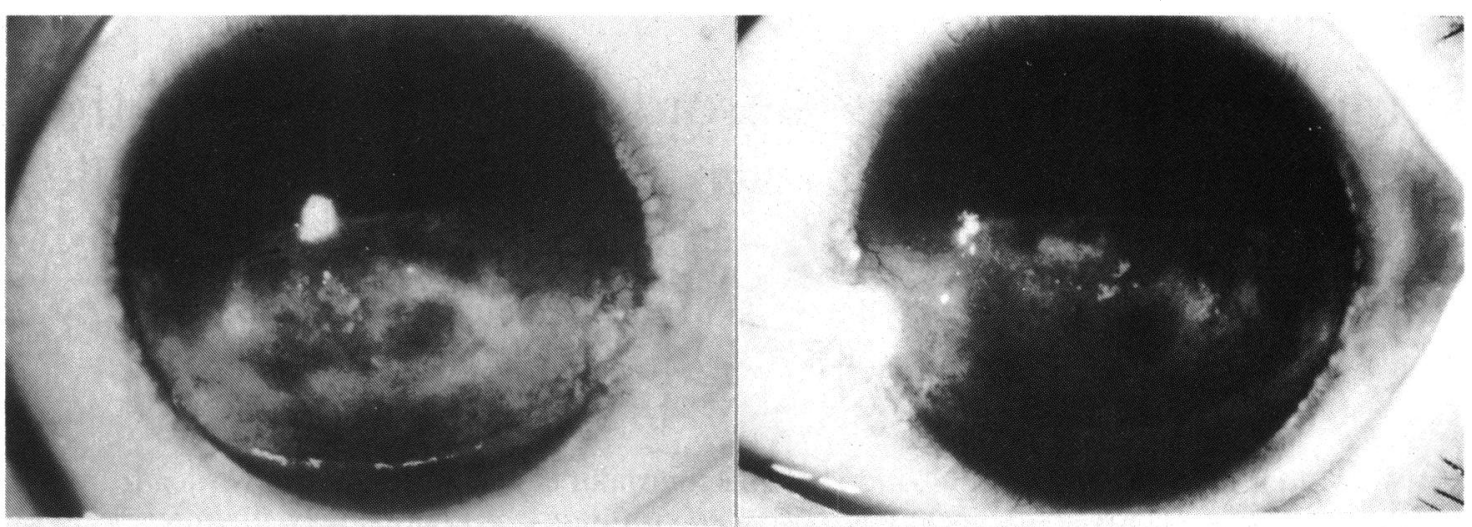

condition in both eyes (Fig. 1, top). Ocular examination revealed corrected visual acuity of $20 / 200$ and intraocular pressure of $12 \mathrm{mmHg}$ bilaterally. Slitlamp examination disclosed symmetrical bandshaped corneal opacities occupying the interpalpebral area in both eyes (Fig. 2, top). These opacities consisted of yellow, globular, oily appearing droplets limited to the anterior stroma. Some globules raised the epithelium in the central cornea. Subepithelial vascular invasion into the opacified area from the nasal, and temporal limbus was observed, as were yellow deposits in the horizontal 
perilimbal conjunctiva. The right eye also contained a nasal pinguecula and the left eye a nasal pterygium. No other ocular abnormality was detected, and general physical and laboratory examinations gave normal results. Rotation autokeratoplasty on the left eye and lamellar keratoplasty on the right eye improved her corrected vision to $20 / 25$ in each eye. The pinguecula of the right eye was also excised at the time of surgery.

CASE 2

A 35-year-old brother of the patient in case 1 was examined in October 1983. His best corrected vision was 20/100 OD and 20/50 OS. Intraocular pressure was $8 \mathrm{mmHg}$ OU. A slit-lamp examination disclosed band-shaped corneal opacities similar to those of case 1 in both eyes (Fig. 2, middle). However, the yellow globules were larger than those of case 1 , especially in the central cornea. Horizontal perilimbal yellow deposits and pingueculae were present bilaterally. His vision had been poor since childhood and had deteriorated slowly. He had also been photophobic during his childhood but had never suffered from painful irritation. Occupational and environmental histories were unremarkable, and in general he had been healthy.

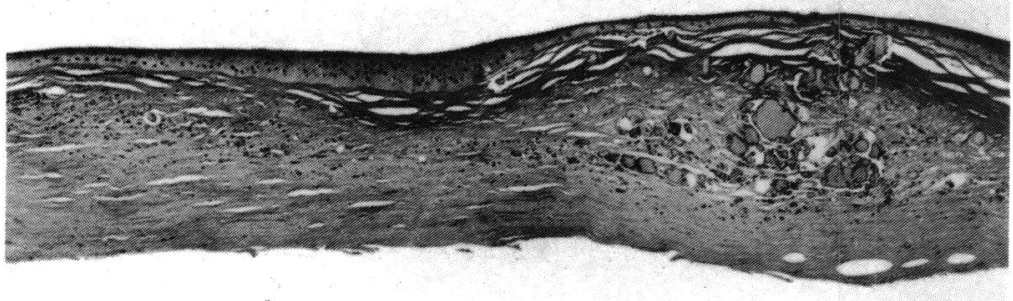

Fig. 3 Histological section of the cornea of case 1. (Top, haematoxylin-eosin, $\times 53$. Bottom, phosphotungstic

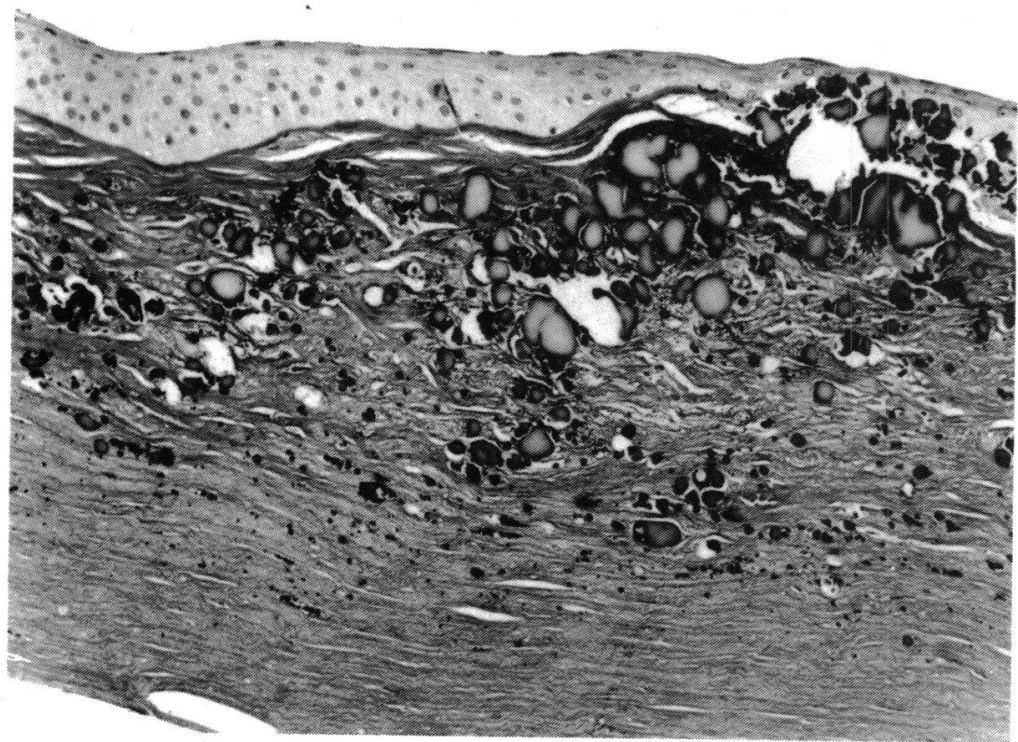
acid/haematoxylin, $\times 132$ ). 
CASE 3

A 35-year-old female was referred for corneal transplant in July 1982 because of slowly progressive blurring of vision and intermittent painful irritation in both eyes for the past 10 years. Her vision had been poor since childhood, and she had a history of photophobia and epiphora as a child. Her parents were first cousins, but no other family member was affected with the same corneal problem (Fig. 2, bottom). Ocular examination revealed corrected visual acuity of 20/200 OD and 20/40 OS. Intraocular pressure was $17 \mathrm{mmHg}$ in each eye. Slit-lamp examination revealed bilateral symmetrical band-shaped corneal opacities occupying the interpalpebral area in both eyes (Fig. 2, bottom). These opacities consisted of fine, yellow, oily appearing globules limited to the anterior stroma. Yellow globular deposits were also observed in the horizontal perilimbal conjunctiva. Subepithelial vascular invasion was detected in every quadrant but was most prominent
Fig. 4 Histological section of the cornea of case 3. (Top, haematoxylin-eosin, $\times 83$. Bottom, Ziehl-Neelsen, $\times 166)$.
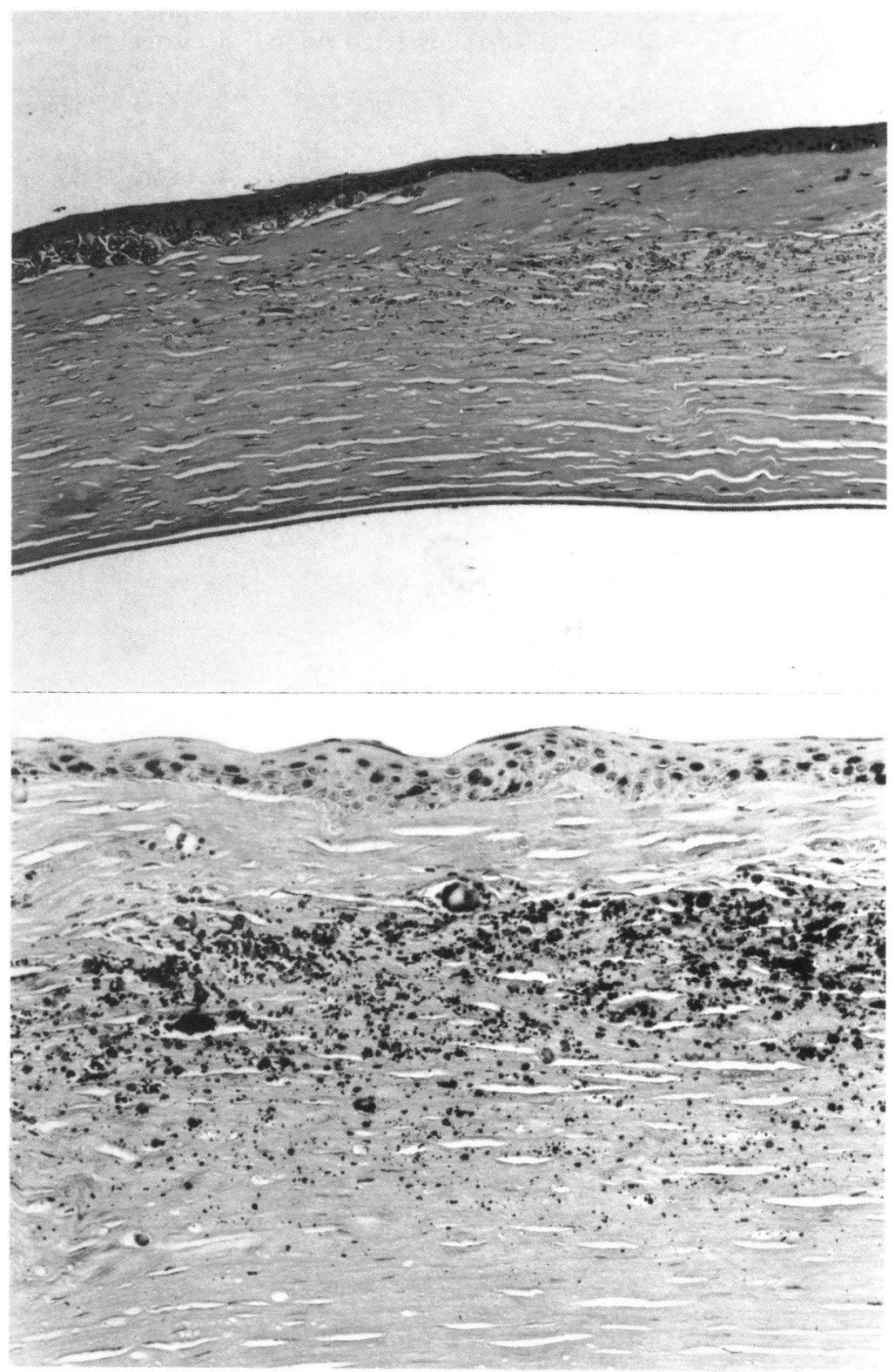
from the horizontal limbus into the opacified area. No other ocular abnormality was detected, and general physical and laboratory examinations were normal. Penetrating keratoplasty was performed on the right eye on 20 August 1982. Allograft rejection occurred one month after surgery but was successfully treated by systemic and local steroid therapy. The corrected vision improved to $20 / 25$ in the right eye.

\section{PATHOLOGICAL FINDINGS}

The histological sections of the corneas from cases 1 and 3 showed eosinophilic globular deposits of various sizes located primarily in the anterior stroma (Figs. 3 and 4, top). These globules stained positively with phosphotungustic acid haematoxylin (Fig. 3, bottom) and Ziehl-Neelsen stains (Fig. 4, bottom). Periodic acid Schiff and luxol fast blue stains were weakly positive. Verhoeff's elastin stain was positive, but this response was unaffected by elastase digestion. Congo red, von Kossa, Mallory's aniline blue, van Gieson elastic, resorcin-fuchsin, orsein, alcian blue, oil red O, Sudan black B, Nile blue, Ralph's method for haemoglobin, and iron stains were all negative. The globules of unstained sections were autofluorescent. Immunohistological studies using the peroxidase anti-peroxidase method showed that these deposits did not react to anti-human-keratin antibody. The epithelium varied in thickness, and in some areas there was remarkable thinning associated with elevation by the globular deposits. Bowman's layer was destroyed, and the superficial stroma had lost the normal parallel arrangement of collagen fibres. Sections of the pinguecula from case 1 showed similar globular deposits in degenerated elastotic subepithelial tissue.

Corneal tissue from case 1 was fixed in $4 \%$ glutaraldehyde and examined electron microscopically. The stromal deposits were markedly electron dense and structureless (Fig. 5). Most of the small globules were round or oval in shape and the larger ones were irregular. They were always extracellular. Some globules showed granularity at their edges, but others had sharp borders. Apparently normal collagen fibrils surrounded some globules, but most were adjacent to degenerating collagen. There was

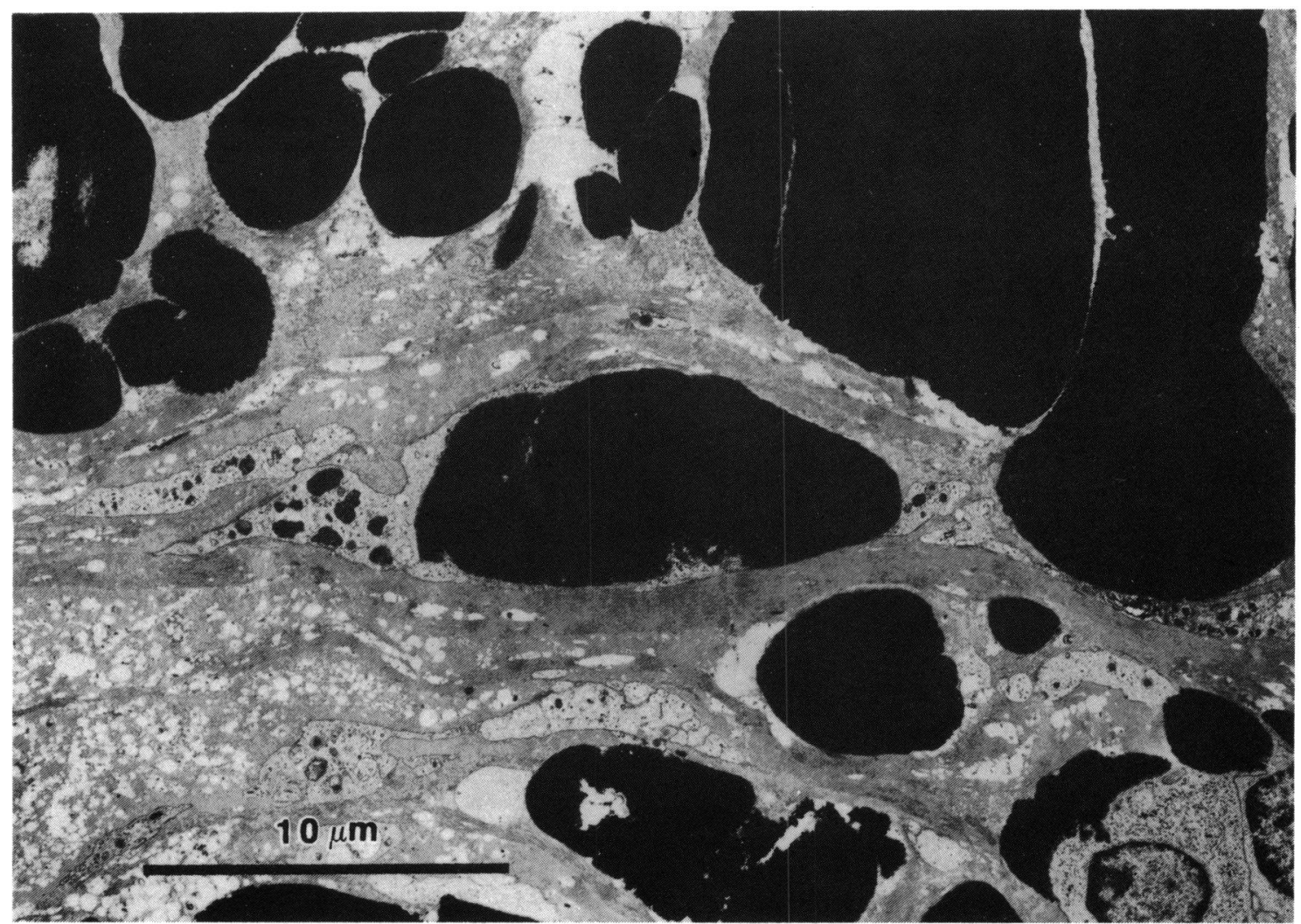

Fig. 5 Transmission electron micrograph of the cornea of case 1 showing the absolutely structureless electron dense deposits of various size in degenerated stroma. $(\times 4200)$. 
also vacuolisation of Bowman's layer and superficial stroma. A few keratocytes contained large electron dense bodies but not any material that resembled the stromal deposits. $X$-ray microanalysis performed with a JEOL 1200EX electron microscope showed no significant differences between the deposited material and the surrounding area.

\section{Discussion}

The corneal degeneration characterised by deposits of yellow, oily appearing globules has been described under a variety of terms, ${ }^{3}$ with spheroidal degeneration being most widely used. There is a variety of situations in which this type of disorder can occur, and complete clinical patterns and histological characteristics are not consistent in previous reports. ${ }^{1-15}$ Nevertheless, there has been agreement that these globular deposits do not constitute a separate disease entity but represent a response of the cornea to various stimuli. ${ }^{3-5}$

Fraunfelder and colleagues ${ }^{13}$ divided this condition into three basic forms: (1) the primary corneal type associated with increasing age; (2) the secondary corneal type associated with significant pre-existing pathology; and (3) the conjunctival type. The primary corneal type in this classification is not a rare occurrence but never involves the central cornea. Our cases cannot be classified by these criteria. All three patients were rather young, had band-shaped depositions involving the central cornea, and had no history of other ocular diseases or of unusual exposures to extreme environments.

Klouček $^{6}$ and Meisler et al. ${ }^{7}$ reported cases of familial band-shaped spheroidal degeneration. Klouček's cases were patients in the fourth and seventh decade but had noted their deterioration of vision in the second or third decade of life. The cases of Meisler $e t$ al. were three brothers, and two of them were in the third decade of life. The changes in the central cornea of the youngest brother were different from the yellow globular depositions. Pedigrees of the families of Meisler et al. and ours suggest an autosomal recessive inheritance of this condition.

Spheroidal degeneration has been reported as a secondary phenomenon in patients with lattice dystrophy, ${ }^{8}$ a primary corneal amyloidosis. Three cases of primary band-shaped keratopathy have been reported in consanguineous families whose relatives had gelatinous drop-like dystrophy, another form of corneal amyloidosis. ${ }^{16-18}$ The clinical and histological findings in these latter cases agree remarkably with ours, though they were not identified as spheroidal degeneration. In one of these three cases of bandshaped keratopathy the patient developed gelatinous drop-like dystrophy only after a superficial keratectomy had been performed. ${ }^{1 \mathrm{x}}$

The aetiology of these globular deposits is controversial. Klintworth ${ }^{4}$ has noted their association with conjunctival elastosis (pingueculae). Two of our cases also had pingueculae or pterygium in both eyes. Garner et al. ${ }^{9}$ demonstrated the pseudoelastic nature of these materials by positive staining with Verhoeff's stain and a lack of response to elastase digestion and other elastic stains. The same staining results were obtained in our cases. The histochemical data from our cases further suggest that the material in the globules has a complex composition, with staining characteristics of both protein and acid-fast lipid. Electron microscopy and $x$-ray analysis of our cases did not reveal any new information about the origin or characteristics of these globules.

\section{References}

1 Fraunfelder FT, Hanna C, Parker JM. Spheroid degeneration of the cornea and conjunctiva. 1. Clinical course and characteristics. Am J Ophthalmol 1972; 74: 821-8.

2 Freedman A. Climatic droplet keratopathy. I. Clinical aspects. Arch Ophthalmol 1973; 89: 193-7.

3 Fraunfelder FT, Hanna C. Spheroidal degeneration of cornea and conjunctiva. 3. Incidence, classification and etiology. Am J Ophthalmol 1973; 76: 41-50.

4 Klintworth GK. Chronic actinic keratopathy. A condition associated with conjunctival elastosis (pinguecula) and typified by characteristic extracellular concretions. Am J Pathol 1972; 67: $327-42$.

5 Brownstein S, Rodrigues MM, Fine BS, Albert EN. The elastotic nature of hyaline corneal deposits. A histochemical, fluorescent, and electron microscopic examination. Am J Ophthalmol 1973; 75: 799-809.

6 Klouček F. Familial band-shaped keratopathy and spheroid degeneration. Clinical and electron microscopic study. Graefges Arch Klin Exp Ophthalmol 1977; 205: 47-59.

7 Meisler DM, Tabara KF, Wood IS, Alvarado JA, Biswell R. Familial band-shaped nodular keratopathy. Ophthalmology (Rochester) 1985; 92: 217-22.

8 Garner A. Keratinoid corneal degeneration. Br J Ophthalmol 1970; 54: 769-80.

9 Garner A, Fraunfelder FT, Barras TC, Hinzpeter, EN. Spheroidal degeneration of cornea and conjunctiva. $\mathrm{Br} J$ Ophthalmol 1976; 60: 473-8.

10 Hanna C, Fraunfelder FT. Spheroid degeneration of the cornea and conjunctiva. 2. Pathology. Am J Ophthalmol 1972; 74: 829-39.

11 Roger FC. Clinical findings, course, and progress of Bietti's corneal degeneration in the Dahlak Islands. Br J Ophthalmol 1973; 57: 657-64.

12 Garner A, Morgan G, Tripathi RC. Climatic droplet keratopathy. II. Pathologic findings. Arch Ophthalmol 1973; 89: 198-204.

13 Johnson GJ, Ghosh M. Labrador keratopathy: clinical and pathological findings. Can J Ophthalmol 1975; 10: 119-35.

14 Rodrigues MM, Laibson PR, Weinerb S. Corneal elastosis Appearance of band-like keratopathy and superficial degeneration. Arch Ophthalmol 1975; 93: 111-4.

15 Christensen GR. Proteinaceous corneal degeneration. A histochemical study. Arch Ophthalmol 1973; 89: 30-2.

16 Kiyosawa M. A familial corneal dystrophy (gelatinous droplike dystrophy). The relationship between hyaline degeneration and 
band-shaped keratopathy. Nippon Ganka Gakkai Zasshi 1932; 36: 1634-45.

17 Kaku K, Suda K. Primary band-shaped and gelatinous dropshaped degeneration of the cornea of a sister and brother.Jpn J Clin Ophthalmol 1964; 18: 807-12.
18 Kanai A, Kaufman HE. Electron microscopic studies of primary band-shaped keratopathy and gelatinous drop-like corneal dystrophy in two brothers. Ann Ophthalmol 1982; 14: 535-9.

Accepted for publication 19 September 1985. 